Tohoku Math. J.

68 (2016), 639-649

\title{
A NOTE ON THE KAKEYA MAXIMAL OPERATOR AND RADIAL WEIGHTS ON THE PLANE
}

\author{
Hiroki SAITO AND Yoshiniro SAwANO
}

(Received October 14, 2014, revised April 6, 2015)

\begin{abstract}
We obtain an estimate of the operator norm of the weighted Kakeya (Nikodým) maximal operator without dilation on $L^{2}(w)$. Here we assume that a radial weight $w$ satisfies the doubling and supremum condition. Recall that, in the definition of the Kakeya maximal operator, the rectangle in the supremum ranges over all rectangles in the plane pointed in all possible directions and having side lengths $a$ and $a N$ with $N$ fixed. We are interested in its eccentricity $N$ with $a$ fixed. We give an example of a non-constant weight showing that $\sqrt{\log N}$ cannot be removed.
\end{abstract}

1. Introduction and Preliminaries. In 1977 A. Córdoba obtained the sharp bound for the Kakeya (Nikodým) maximal operator, which is related to many important problems in Fourier analysis. Recall that the Kakeya (Nikodým) maximal operator is equipped with a sufficiently large natural number $N$ and positive real number $a$ and that this is given as follows: Let $\mathcal{B}_{N}^{a}$ be the set of all rectangles in $\mathbb{R}^{n}$ which are pointed to all possible directions and are congruent with $[0, a]^{n-1} \times[0, a N]$. The Kakeya (Nikodým) maximal operator without dilation $K_{N}^{a}$ is defined by

$$
K_{N}^{a} f(x):=\sup _{x \in R \in \mathcal{B}_{N}^{a}} \frac{1}{|R|} \int_{R}|f(y)| d y, \quad \text { for } f \in L_{\text {loc }}^{1}\left(\mathbb{R}^{n}\right),
$$

where the supremum is taken over all rectangles $R \in \mathcal{B}_{N}^{a}$ containing $x \in \mathbb{R}^{n}$. The Kakeya (Nikodým) maximal operator is defined by

$$
K_{N} f(x):=\sup _{a>0} K_{N}^{a} f(x) .
$$

It is conjectured that $K_{N}$ is bounded on $L^{n}\left(\mathbb{R}^{n}\right)$ with the norm which grows no faster than $O\left((\log N)^{\alpha_{n}}\right)$ for some $\alpha_{n}>0$ as $N \rightarrow \infty$. When $n=2$, the optimal bound

$$
\left\|K_{N}^{a} f\right\|_{L^{2}\left(\mathbb{R}^{2}\right)} \leq C \sqrt{\log N}\|f\|_{L^{2}\left(\mathbb{R}^{2}\right)}
$$

was proved by Córdoba [5, Proposition 1.2]. In this paper, we shall carry over this result to a weighted setting. So, we place ourselves in the setting of $\mathbb{R}^{n}$ with $n \geq 2$ equipped with a weighted measure $w d x$, where $w: \mathbb{R}^{n} \rightarrow(0, \infty)$ is a measurable function. In the higher

2010 Mathematics Subject Classification. Primary 42B25.

Key words and phrases. Kakeya maximal operator, radial weight.

The second author was supported by Grant-in-Aid for Young Scientists (B), No. 24740085, Japan Society for the Promotion of Science. 
dimensional case, $n>2$, (1.1) is still open in general; (1.1) was proved only for some special classes of functions.

Before we describe the conditions on weights, let us recall some related definitions. A more powerful but complicated maximal operator has been studied on the plane. Strömberg studied the directional maximal operator defined to be

$$
M_{\Omega} f\left(x_{1}, x_{2}\right):=\sup _{r \in \mathbb{R}^{+},\left(v_{1}, v_{2}\right) \in \Omega} \frac{1}{2 r} \int_{-r}^{r}\left|f\left(x_{1}+t v_{1}, x_{2}+t v_{2}\right)\right| d t \quad\left(x_{1}, x_{2}\right) \in \mathbb{R}^{2},
$$

where $\Omega$ is the set of unit vectors in $\mathbb{R}^{2}$ of cardinality $N$. He proved that

$$
\left\|M_{\Omega} f\right\|_{L^{2}\left(\mathbb{R}^{2}\right)} \leq C \log N\|f\|_{L^{2}\left(\mathbb{R}^{2}\right)},
$$

when $\Omega$ is equidistributed [15, Theorem 1(2)]. Moreover, a geometrical observation shows

$$
K_{N} f(x) \leq C M_{\Omega} f(x) \quad\left(x \in \mathbb{R}^{n}\right),
$$

if $\Omega$ is equidistributed. Note that (1.3) and (1.4) yield

$$
\left\|K_{N} f\right\|_{L^{n}\left(\mathbb{R}^{n}\right)} \leq C(\log N)^{\alpha_{n}}\|f\|_{L^{n}\left(\mathbb{R}^{n}\right)}
$$

with $\alpha_{n}=1$ and $n=2$. Katz established (1.3) without assuming that $\Omega$ is an equidistributed set of directions; see [9, Theorem 1] for (1.5) with $\alpha_{n}=1$. Alfonseca, Soria and Vargas [1, 2] studied $M_{\Omega}$ by using a geometrical method and they simplified Katz's proof. The first author and H. Tanaka [13] discussed the weighted version of $M_{\Omega}$ by the method of [1, 2], and extended Katz's result for the power weight $w$, i.e., $w(x)=|x|^{\lambda}$ for any $\lambda>0$. We will explain this more precisely. Let $L^{2}(w)$ be the weighted $L^{2}$ space of all measurable functions $f$ for which the norm

$$
\|f\|_{L^{2}(w)}:=\left(\int_{\mathbb{R}^{n}}|f(x)|^{2} w(x) d x\right)^{\frac{1}{2}}
$$

is finite. The weighted Kakeya maximal operator is defined to be

$$
K_{N, w} f(x):=\sup _{x \in R \in \mathcal{B}_{N}} \frac{1}{w(R)} \int_{R}|f(y)| w(y) d y \quad\left(x \in \mathbb{R}^{n}\right),
$$

where $w(R):=\int_{R} w(x) d x$. The weighted directional maximal operator $M_{\Omega, w}$ is defined analogously starting from the definition (1.2). In a similar fashion, we can verify

$$
K_{N, w} f(x) \leq C M_{\Omega, w} f(x) \quad\left(x \in \mathbb{R}^{n}\right),
$$

if $\Omega$ is equidistributed. The result in [13] together with (1.7) implies

$$
\left\|K_{N, w} f\right\|_{L^{2}(w)} \leq C \log N\|f\|_{L^{2}(w)} .
$$

In a similar way, we define the weighted Kakeya maximal operator without dilation by

$$
K_{N, w}^{a} f(x):=\sup _{x \in R \in \mathcal{B}_{N}^{a}} \frac{1}{w(R)} \int_{R}|f(y)| w(y) d y
$$

for $a>0$, so that

$$
K_{N, w} f=\sup _{a>0} K_{N, w}^{a} f
$$


for all measurable functions $f$. By (1.8) and (1.9), we have immediately the estimate $\left\|K_{N, w}^{a} f\right\|_{L^{2}(w)} \leq C \log N\|f\|_{L^{2}(w)}$. However, unfortunately this estimate is not sharp. In this paper, we improve the upper bound of the operator norm of $K_{N, w}^{a}: L^{2}(w) \rightarrow L^{2}(w)$. Moreover, we can consider slightly general radial weights, not necessarily power weight, satisfying the following two conditions:

Doubling condition: For all $0 \leq r_{1} \leq r_{1}^{\prime} \leq r_{2}^{\prime} \leq r_{2}<\infty$ with $r_{2}-r_{1}=2\left(r_{2}^{\prime}-r_{1}^{\prime}\right)$,

$$
\int_{r_{1}}^{r_{2}} w_{0}(r) d r \leq C \int_{r_{1}^{\prime}}^{r_{2}^{\prime}} w_{0}(r) d r ;
$$

Supremum condition: For all $0<r_{1}<r_{2}<\infty$,

$$
\sup _{r_{1}<r<r_{2}} w_{0}(r) \leq \frac{C}{r_{2}-r_{1}} \int_{r_{1}}^{r_{2}} w_{0}(r) d r .
$$

We shall show the following estimate:

THEOREM 1.1. Assume that $w$ satisfies the doubling condition (1.10) and the supremum condition (1.11). Then there exists a constant $C$ which depends only on constants $C$ in (1.10) and (1.11) such that

$$
\left\|K_{N, w}^{a} f\right\|_{L^{2}(w)} \leq C \sqrt{\log N}\|f\|_{L^{2}(w)}
$$

for all $N \geq 2, a>0$ and $f \in L^{2}(w)$.

To pass to our weighted case, the following lemma, which is used in the proof of the weighted maximal operator in [13], is indispensable. For an $A \subset \mathbb{R}^{2}$, we denote by $\operatorname{rad}(A)$ an interval (inf $\operatorname{inA}_{x \in A}|x|, \sup _{x \in A}|x|$ ). We have obtained the following lemma in [13]. Here and below, to denote the inequality $X \leq C Y$, where $C$ is a positive constant independent of main parameters and functions. By $X \lesssim Y$ we mean $X \leq C Y$ for some constant $C$. We write $X \sim Y$ if both $X \lesssim Y$ and $Y \lesssim X$ are true. Keeping these notations in mind, we invoke our key lemma from [13].

LEMMA 1.2. Let $R \subset \mathbb{R}^{2}$ be a rectangle, and let $w_{0}$ satisfy (1.10) and (1.11). Then

$$
w(R) \sim \frac{|R|}{|\operatorname{rad}(R)|} \int_{\operatorname{rad}(R)} w_{0}(r) d r .
$$

Here we recall some related estimates. More is known when $f$ is a function of product type or radial type. In [8, Theorem], Igari obtained (1.5) with $\alpha_{n}=3 / 2$ and $n \geq 2$ for all functions of product type. H. Tanaka improved Igari's result in [16]; $\alpha_{n}=1-1 / n$ suffices.

Next, we will show that our bound is the optimal, i.e., it is natural to ask ourselves whether there exists a function $\tilde{f}$ such that

$$
\left\|K_{N, w}^{a} \tilde{f}\right\|_{L^{2}(w)} \geq C \sqrt{\log N}\|\tilde{f}\|_{L^{2}(w)}
$$

holds and we answer this question in the affirmative. 
By using the function $f_{N}(x)=\frac{1}{|x|} \chi_{\{1 \leq|x| \leq N\}}(x)$, in [12, Lemma 1], Müller checked that (1.1) is optimal. He actually placed himself in $\mathbb{R}^{n}$ and showed

$$
\left\|K_{N}^{a} f_{N}\right\|_{L^{n}\left(\mathbb{R}^{n}\right)} \geq C(\log N)^{\frac{n-1}{n}}\left\|f_{N}\right\|_{L^{n}\left(\mathbb{R}^{n}\right)} .
$$

See also the textbook [7, Proposition 10.3.4].

We would like to thank Professor Joonil Kim who motivated this study. We also express deep gratitude to a suggestion on Section 3 by Professor Neal Bez. We thank anonymous referee for his (or her) helpful comments.

2. Proof of Theorem 1.1. We start with the reduction. First, we may assume that $f$ is nonnegative and essentially bounded. Write $f_{a}(x)=f(a x)$ and $w_{a}(x)=w(a x)$ for $a>0$. Observe that the constants in (1.10) and (1.11) for $w$ and those for $w_{a}$ can be taken the same. By a scaling argument, we can verify

$$
\frac{\left\|K_{N, w}^{a} f\right\|_{L^{2}(w)}}{\|f\|_{L^{2}(w)}}=\frac{\left\|K_{N, w_{a}}^{1} f_{a}\right\|_{L^{2}\left(w_{a}\right)}}{\left\|f_{a}\right\|_{L^{2}\left(w_{a}\right)}},
$$

so that it is suffices to consider $a=1$. We divide $\mathbb{R}^{2}$ into open unit squares $Q_{\alpha}$ which have center at lattice point $\alpha \in \mathbb{Z}^{2}$ and whose sides are parallel to the axes, so that

$$
\sum_{\alpha \in \mathbb{Z}^{2}} \chi_{Q_{\alpha}}=1
$$

almost everywhere. By the essential boundedness of $f$, for every square $Q_{\alpha}$, we can find a rectangle $R_{\alpha} \in \mathcal{B}_{N}^{1}$ such that $Q_{\alpha} \cap R_{\alpha} \neq \emptyset$ and

$$
K_{N, w}^{1} f(x) \leq \frac{2}{w\left(R_{\alpha}\right)} \int_{R_{\alpha}} f(y) w(y) d y \quad \text { for } x \in Q_{\alpha} .
$$

Then, it suffices for the proof of the theorem 1.1 to estimate

$$
T_{w} f(x):=\sum_{\alpha \in \mathbb{Z}^{2}}\left(\frac{1}{w\left(R_{\alpha}\right)} \int_{R_{\alpha}} f w\right) \chi_{Q_{\alpha}}(x) \text { for } f \in L^{2}(w)
$$

by using the bounds which are independent of the $R_{\alpha}$ 's.

For the proof, we need two lemmas. The first one is a simple geometric argument.

LEMMA 2.1. For all $\gamma \in \mathbb{Z}^{2}$

$$
\sum_{\alpha \in \mathbb{Z}^{2}}\left|R_{\alpha} \cap R_{\gamma}\right| \lesssim N^{2} \log N
$$

This is essentially due to Córdoba [5, p. 7 line 4] and also closely related to [12, Lemma 2]. For reader's convenience, we recall the proof of (2.1) in Section 4.

The following lemma is originally due to Carbery in [3, Theorem 2]:

LEMMA 2.2. Let $T_{w}$ be as above. Then $T_{w}$ is of strong type $(p, p)$ with respect to the weighted measure $w(x) d x$ if and only if there exists a constant $C_{q}$, such that for any sequence 
$\left\{\lambda_{\alpha}\right\} \subset \mathbb{R}_{+}$, we have

$$
\int\left(\sum_{\alpha \in \mathbb{Z}^{2}} \lambda_{\alpha} \frac{w\left(Q_{\alpha}\right)}{w\left(R_{\alpha}\right)} \chi_{R_{\alpha}}(x)\right)^{q} w(x) d x \leq C_{q} \sum_{\alpha \in \mathbb{Z}^{2}} \lambda_{\alpha}^{q} w\left(Q_{\alpha}\right),
$$

where $q$ is the conjugate of $p$. Moreover, the infimum of the constants $\left(C_{q}\right)^{1 / q}$ satisfying (2.2) is $\left\|T_{w}\right\|_{L^{p}(w) \rightarrow L^{p}(w)}$.

PROOF. We go through the same argument as the proof of [2, Theorem 3]. set

By Lemma 2.2 with $p=q=2$ it is sufficient to show (2.2) with $C_{2}=O(\log N)$. We

$$
I:=\sqrt{\int_{\mathbb{R}^{2}}\left(\sum_{\alpha \in \mathbb{Z}^{2}} \lambda_{\alpha} \frac{w\left(Q_{\alpha}\right)}{w\left(R_{\alpha}\right)} \chi_{R_{\alpha}}(x)\right)^{2} w(x) d x .}
$$

Calculating the integral, we obtain

$$
\begin{aligned}
I^{2} & =\sum_{\alpha \in \mathbb{Z}^{2}} \sum_{\beta \in \mathbb{Z}^{2}} \lambda_{\alpha} \lambda_{\beta} \frac{w\left(Q_{\alpha}\right) w\left(Q_{\beta}\right)}{w\left(R_{\alpha}\right) w\left(R_{\beta}\right)} w\left(R_{\alpha} \cap R_{\beta}\right) \\
& =\sum_{\alpha \in \mathbb{Z}^{2}} \sum_{\beta \in \mathbb{Z}^{2}} \lambda_{\alpha} \lambda_{\beta} \frac{\sqrt{w\left(Q_{\alpha}\right) w\left(Q_{\beta}\right)}}{w\left(R_{\alpha}\right) w\left(R_{\beta}\right)} w\left(R_{\alpha} \cap R_{\beta}\right) \cdot \sqrt{w\left(Q_{\alpha}\right) w\left(Q_{\beta}\right)} .
\end{aligned}
$$

From Lemma 1.2, we deduce that

$$
\begin{aligned}
& \frac{\sqrt{w\left(Q_{\alpha}\right) w\left(Q_{\beta}\right)}}{w\left(R_{\alpha}\right) w\left(R_{\beta}\right)} w\left(R_{\alpha} \cap R_{\beta}\right) \\
& \sim \frac{1}{N^{2}} \frac{\left(\frac{1}{\left|\operatorname{rad}\left(Q_{\alpha}\right)\right|} \int_{\operatorname{rad}\left(Q_{\alpha}\right)} w_{0}\right)^{\frac{1}{2}}}{\frac{1}{\left|\operatorname{rad}\left(R_{\alpha}\right)\right|} \int_{\operatorname{rad}\left(R_{\alpha}\right)} w_{0}} \cdot \frac{\left(\frac{1}{\left|\operatorname{rad}\left(Q_{\beta}\right)\right|} \int_{\operatorname{rad}\left(Q_{\beta}\right)} w_{0}\right)^{\frac{1}{2}}}{\frac{1}{\left|\operatorname{rad}\left(R_{\beta}\right)\right|} \int_{\operatorname{rad}\left(R_{\beta}\right)} w_{0}} \cdot \frac{\left|R_{\alpha} \cap R_{\beta}\right|}{\left|\operatorname{rad}\left(R_{\alpha} \cap R_{\beta}\right)\right|} \int_{\operatorname{rad}\left(R_{\alpha} \cap R_{\beta}\right)} w_{0} \\
& \lesssim \frac{1}{N^{2}} \frac{\left(\sup _{\operatorname{rad}\left(Q_{\alpha}\right)} w_{0}\right)^{\frac{1}{2}}}{\sup _{\operatorname{rad}\left(R_{\alpha}\right)} w_{0}} \cdot \frac{\left(\sup _{\operatorname{rad}\left(Q_{\beta}\right)} w_{0}\right)^{\frac{1}{2}}}{\sup _{\operatorname{rad}\left(R_{\beta}\right)} w_{0}} \cdot\left|R_{\alpha} \cap R_{\beta}\right| \sup _{\operatorname{rad}\left(R_{\alpha} \cap R_{\beta}\right)} w_{0},
\end{aligned}
$$

where we have used the supremum condition in the last inequality and $\left|Q_{\alpha}\right|=1$. Since $Q_{\alpha} \subset 2 R_{\alpha}$ for any $\alpha$, we have $\operatorname{rad}\left(Q_{\alpha}\right) \subset \operatorname{rad}\left(2 R_{\alpha}\right)$. By the doubling condition of $w_{0}$,

$$
\begin{aligned}
& \frac{\sqrt{w\left(Q_{\alpha}\right) w\left(Q_{\beta}\right)}}{w\left(R_{\alpha}\right) w\left(R_{\beta}\right)} w\left(R_{\alpha} \cap R_{\beta}\right)
\end{aligned}
$$

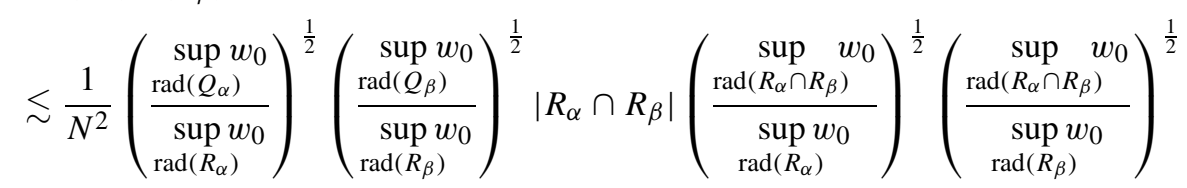




$$
\lesssim \frac{1}{N^{2}}\left|R_{\alpha} \cap R_{\beta}\right|
$$

Therefore, we have

$$
I^{2} \lesssim \frac{1}{N^{2}} \sum_{\alpha \in \mathbb{Z}^{2}} \sum_{\beta \in \mathbb{Z}^{2}} \lambda_{\alpha} \lambda_{\beta} \sqrt{w\left(Q_{\alpha}\right) w\left(Q_{\beta}\right)}\left|R_{\alpha} \cap R_{\beta}\right| .
$$

Therefore, from (2.1), we have

$$
\begin{aligned}
I^{2} & \lesssim \frac{1}{N^{2}} \cdot \sum_{\alpha \in \mathbb{Z}^{2}} \sum_{\beta \in \mathbb{Z}^{2}} \lambda_{\alpha} \lambda_{\beta} \sqrt{w\left(Q_{\alpha}\right) w\left(Q_{\beta}\right)}\left|R_{\alpha} \cap R_{\beta}\right| \\
& \leq \frac{1}{N^{2}} \cdot \frac{1}{2}\left\{\sum_{\alpha \in \mathbb{Z}^{2}} \lambda_{\alpha}^{2} w\left(Q_{\alpha}\right)\left(\sum_{\beta \in \mathbb{Z}^{2}}\left|R_{\alpha} \cap R_{\beta}\right|\right)+\sum_{\beta \in \mathbb{Z}^{2}} \lambda_{\beta}^{2} w\left(Q_{\beta}\right)\left(\sum_{\alpha \in \mathbb{Z}^{2}}\left|R_{\alpha} \cap R_{\beta}\right|\right)\right\} \\
& \lesssim \frac{1}{N^{2}} \cdot \frac{1}{2}\left\{N^{2} \log N \sum_{\alpha \in \mathbb{Z}^{2}} \lambda_{\alpha}^{2} w\left(Q_{\alpha}\right)+N^{2} \log N \sum_{\beta \in \mathbb{Z}^{2}} \lambda_{\beta}^{2} w\left(Q_{\beta}\right)\right\} \\
& =\log N \sum_{\alpha \in \mathbb{Z}^{2}} \lambda_{\alpha}^{2} w\left(Q_{\alpha}\right),
\end{aligned}
$$

where we have used the inequality of arithmetic mean and geometric mean in the second inequality, and also we have used (2.1) in the third inequality. This completes the proof.

REMARK 2.3. By definition it is easy to see that

$$
T_{w} f(x) \leq K_{N, w}^{1} f(x)
$$

for any choice of rectangles $\left\{R_{\alpha}\right\}$. This technique is known as the linearization of sublinear operators.

3. Sharpness of the operator norms in some special cases. In this section, we shall prove that the bound of Theorem 1.1 is optimal for the weights $w(x)=|x|^{\lambda}$, and $w(x)=$ $\log (1+|x|), x \in \mathbb{R}^{2}$, where $\lambda>0$ is a fixed parameter. It is easily seen that these weights satisfy the doubling and supremum conditions (1.10) and (1.11).

3.1. Case $w(x)=|x|^{\lambda}, \lambda>0$. Before starting to prove, we introduce some notations due to Keich [11]. Let $l$ be a line segment $l:=\{(x, a x+b): x \in[0,1]\}$. For such a line $l$, we define $l(x)=a x+b$ and put $a(l):=a$ and $b(l):=b$. We consider lines such that $a(l) \in[0,1]$ and $b(l) \in[-1,0]$. For $\delta>0$ and such an $l$, let $R_{\delta}(l)$ be the triangle defined by the vertices $\{(0, l(0)),(0, l(0)-\delta),(1, l(1))\}$. Let $\vec{R}_{\delta}(l)$ be the triangle obtained by translating $R_{\delta}(l)$ by $2 \sqrt{2}$ along the (positive) direction of $l$. In words of the book [14, p. 435], we can say that $\vec{R}_{\delta}(l)$ is a reach of $R_{\delta}(l)$. We use the following theorem:

THEOREM 3.1 [11, Theorem 1]. For any $n$, there exists a collection of $2^{n}$ line segments $\left\{l_{i}^{n}: i=0,1,2, \ldots, 2^{n}-1\right\}$ such that the triangles $R_{2^{-n}}\left(l_{i}^{n}\right)$ satisfy the following two properties:

(1) $a\left(l_{i}^{n}\right)=i 2^{-n}$. 
(2) $\left|\bigcup_{i=0}^{2^{n}-1} R_{2^{-n}}\left(l_{i}^{n}\right)\right|<\frac{1}{n}$.

(3) The translated triangles, $\vec{R}_{2^{-n}}\left(l_{i}^{n}\right)$ are disjoint.

See $[14$, p. 435, Theorem 1] for a similar construction.

Let

$$
E_{n}:=\bigcup_{i=0}^{2^{n}-1} R_{2^{-n}}\left(l_{i}^{n}\right) .
$$

Then $E_{n}$ has a unit length line segment with any given slope $a \in[0,1]$, it is composed of triangles with area $2^{-n}$, and $\left|E_{n}\right|<1 / n$. Moreover, it should be noted that we can construct the union of such triangles $R_{2^{-n}}\left(l_{i}^{n}\right)$ and $\vec{R}_{2^{-n}}\left(l_{i}^{n}\right)$ in a compact disc $D$, independent of $n$, which does not contain the origin.

COROLlary 3.2. For the power weight $w(x)=|x|^{\lambda}$, Theorem 1.1 is optimal, that is,

$$
\left\|K_{N, w}^{1 / N}\right\|_{L^{2}(w) \rightarrow L^{2}(w)} \gtrsim \sqrt{\log N} .
$$

Proof. For fixed $N, N \gg 1$, let $n \in \mathbb{N}$ be $2^{n} \sim N$, and $a=1 / N$. Applying Theorem 3.1 to $n$, we get the family of rectangles $\left\{R_{2^{-n}}\left(l_{i}^{n}\right)\right\}$ such that

$$
\left|E_{n}\right|=\left|\bigcup_{i=0}^{2^{n}-1} R_{2^{-n}}\left(l_{i}^{n}\right)\right| \lesssim \frac{1}{\log N} .
$$

We can find a compact disc $D$, containing these triangles and translated ones, independent of $n$. The weight $w(x)=|x|^{\lambda}$ can be regard as a constant function on $D$ so that we see that $\|\cdot\|_{L^{2}(w)} \sim\|\cdot\|_{L^{2}\left(\mathbb{R}^{2}\right)}$ and $K_{N, w}^{1 / N} f(x) \sim K_{N}^{1 / N} f(x)$. Let $f_{n}:=\chi_{E_{n}}$. Then by (i) of Theorem 3.1,

$$
\left\|f_{n}\right\|_{L^{2}(w)}^{2} \sim\left\|f_{n}\right\|_{L^{2}\left(\mathbb{R}^{2}\right)}^{2}=\left|E_{n}\right| \lesssim \frac{1}{\log N} .
$$

On the other hand, let $\widetilde{K}_{N, w}=K_{3 \sqrt{2} N, w}^{1 / N}$ and $\widetilde{K}_{N}=K_{3 \sqrt{2} N}^{1 / N}$, respectively. Then we can verify easily that

$$
\widetilde{K}_{N, w} f_{n}(x) \sim \widetilde{K}_{N} f_{n}(x) \gtrsim 1
$$

for all $x \in \bigcup_{i=1}^{2^{n}-1} \vec{R}_{2^{-n}}\left(l_{i}^{n}\right)$. Since $\vec{R}_{2^{-n}}\left(l_{i}^{n}\right)$ are disjoint, it follows that

$$
\begin{aligned}
\left\|\widetilde{K}_{N, w} f_{n}\right\|_{L^{2}(w)}^{2} & =\int_{\mathbb{R}^{2}} \widetilde{K}_{N, w} f_{n}(x)^{2} w(x) d x \\
& \gtrsim \int_{\bigcup \vec{R}_{2^{-n}}\left(l_{i}^{n}\right)} \widetilde{K}_{N} f_{n}(x)^{2} d x \\
& \gtrsim\left|\bigcup_{i=0}^{2^{n}-1} \vec{R}_{2^{-n}}\left(l_{i}^{n}\right)\right| \sim \sum_{i=0}^{2^{n}-1}\left|\vec{R}_{2^{-n}}\left(l_{i}^{n}\right)\right|=1 .
\end{aligned}
$$


Therefore we have

$$
\frac{\left\|K_{N, w}^{1 / N} f_{n}\right\|_{L^{2}(w)}^{2}}{\left\|f_{n}\right\|_{L^{2}(w)}^{2}} \gtrsim \log N
$$

3.2. Case $w(x)=\log (1+|x|)$. For the moment, we assume that $f \in L^{2}(w)$ is radial, i.e., $f(x)=f_{0}(|x|)$ for some $f_{0}$, and $f$ is supported on an annulus $\mathcal{A}_{N}=\left\{x \in \mathbb{R}^{2}: 1 \leq\right.$ $|x| \leq N\}$. Then by polar coordinates,

$$
\|f\|_{L^{2}(w)}^{2}=\int_{\mathcal{A}_{N}} f(x)^{2} w(x) d x=2 \pi \int_{1}^{N} f_{0}(r)^{2} w_{0}(r) r d r .
$$

On the other hand, if $x \in \mathcal{A}_{N}$, as in [7, 12], we choose a rectangle

$$
R=[0, N] \times\left[-\frac{1}{2}, \frac{1}{2}\right]
$$

in $\mathcal{B}_{N}^{1}$ whose short-side contains origin. Thus,

$$
\begin{aligned}
K_{N, w}^{1} f(x) & \geq \frac{1}{w(R)} \int_{R \cap \mathcal{A}_{N}} f(x) w(x) d x \\
& \geq \frac{1}{w(R)} \int_{2}^{N} \int_{0}^{\sin ^{-1} \frac{1}{2 r}} f_{0}(r) w_{0}(r) r d \theta d r \\
& \geq \frac{1}{2 w(R)} \int_{2}^{N} f_{0}(r) w_{0}(r) d r
\end{aligned}
$$

for all $x \in R \cap \mathcal{A}_{N}$. Due to the rotational invariance of $K_{N, w}^{1} f(x)$,

$$
K_{N, w}^{1} f(x) \geq \frac{1}{2 w(R)} \int_{2}^{N} f_{0}(r) w_{0}(r) d r
$$

for all $x \in \mathcal{A}_{N}$, and hence

$$
\left\|K_{N, w}^{1} f\right\|_{L^{2}(w)}^{2} \geq \frac{w\left(\mathcal{A}_{N}\right)}{4 w(R)^{2}}\left(\int_{2}^{N} f_{0}(r) w_{0}(r) d r\right)^{2} .
$$

Therefore, we have the following formula:

$$
\frac{\left\|K_{N, w}^{1} f\right\|_{L^{2}(w)}^{2}}{\|f\|_{L^{2}(w)}^{2}} \gtrsim \frac{w\left(\mathcal{A}_{N}\right)}{w(R)^{2}} \cdot \frac{\left(\int_{2}^{N} f_{0}(r) w_{0}(r) d r\right)^{2}}{\int_{1}^{N} f_{0}(r)^{2} w_{0}(r) r d r} .
$$

COROllary 3.3. For the weight $w(x)=\log (1+|x|)$, Theorem 1.1 is optimal, that is,

$$
\left\|K_{N, w}^{1}\right\|_{L^{2}(w) \rightarrow L^{2}(w)} \gtrsim \sqrt{\log N} .
$$


We consider $w_{0}(r)=\log (1+r)$ in the formula (3.1). By simple calculation, we have

$$
w\left(\mathcal{A}_{N}\right) \sim N^{2} \log N, \quad w(R) \sim N \log N .
$$

Now we choose $f_{0}(r)=\frac{1}{r}$, then

$$
\begin{aligned}
\frac{\left\|K_{N, w}^{1} f\right\|_{L^{2}(w)}^{2}}{\|f\|_{L^{2}(w)}^{2}} & \gtrsim \frac{N^{2} \log N}{(N \log N)^{2}} \cdot \int_{1}^{N} \frac{1}{r} \log (1+r) d r \\
& \geq \frac{1}{\log N} \int_{1}^{N} \frac{1}{1+r} \log (1+r) d r=\frac{(\log (1+N))^{2}}{2 \log N} \sim \log N .
\end{aligned}
$$

We conclude this paper with related examples.

REMARK 3.4.

(1) In particular, when $w_{0} \equiv 1$, choosing $f_{0}=\frac{1}{r}$, we recover the classical result from (3.1):

$$
\frac{\left\|K_{N}^{1} f\right\|_{L^{2}\left(\mathbb{R}^{2}\right)}^{2}}{\|f\|_{L^{2}\left(\mathbb{R}^{2}\right)}^{2}} \gtrsim \log N .
$$

(2) Consider $w_{0}(r)=r^{\lambda}$ for $\lambda>0$, which corresponds to the power weight $|x|^{\lambda}$. We observe

$$
w\left(\mathcal{A}_{N}\right) \sim N^{\lambda+2}, \quad w(R) \sim N^{\lambda+1} .
$$

If we apply (3.1) to $f_{0}=\frac{1}{r}$, it follows that

$$
\frac{\left\|K_{N, w}^{1} f\right\|_{L^{2}(w)}^{2}}{\|f\|_{L^{2}(w)}^{2}} \gtrsim \frac{1}{N^{\lambda}} \cdot \frac{\left(\int_{1}^{N} r^{\lambda-1} d r\right)^{2}}{\int_{1}^{N} r^{\lambda-1} d r} \sim 1 .
$$

This means that the classical example, $f(x)=\frac{1}{|x|}$ supported on $\mathcal{A}_{N}$, does not work to get optimality for the power weight $w(x)=|x|^{\lambda}$. Moreover, if we substitute $f_{0}(r)=1 / r^{a}$ to the right-hand side (3.1), then a simple calculation gives us

$$
\text { R.H.S.(3.1) } \sim \frac{1}{N^{\lambda}} \cdot \frac{\left(\int_{1}^{N} r^{\lambda-a} d r\right)^{2}}{\int_{1}^{N} r^{\lambda+1-2 a} d r} \sim \begin{cases}\frac{(\log N)^{2}}{N^{\lambda}} & (a=\lambda+1), \\ 1 / \log N & \left(a=\frac{\lambda+2}{2}\right), \\ 1 & \text { (otherwise) } .\end{cases}
$$

Therefore, we cannot obtain the $\sqrt{\log N}$ growth for any $a \in \mathbb{R}$.

(3) It is natural to expect that the bound of Theorem 1.1 is optimal for all weights $w$ satisfying (1.10) and (1.11). But this seems subtler and it is an open problem.

(4) In this paper, we handle the weighted Kakeya maximal operator without dilation $K_{N, w}^{a}$ only. It is natural to ask ourselves what happens for the (ordinal) weighted 
Kakeya maximal operator $K_{N, w}$, when $w$ satisfies the doubling and supremum conditions. However, we leave the matters to the forthcoming paper because it needs a different approach.

4. Proof of (2.1). In this section we will prove

$$
\sum_{\gamma \in \mathbb{Z}^{2}}\left|R_{\alpha} \cap R_{\gamma}\right| \lesssim N^{2} \log N, \quad \text { for each } \alpha \in \mathbb{Z}^{2} .
$$

We begin with the following lemma:

LEMMA 4.1. Let $R_{1}$ and $R_{2}$ be infinite strips with width 1 and $\theta$ be the angle of their long sides. Then

$$
\left|R_{1} \cap R_{2}\right|=\frac{1}{\sin \theta} .
$$

The proof of this lemma is not hard and the proof is omitted. With this lemma in mind, we prove (2.1).

We start with some reductions and some notations. By the translation invariance, we may suppose that $\alpha=0$. Denote by $\theta_{0} \in[0, \pi)$ the angle of the long side of $R_{0}$ and the $x$-axis. By the change of coordinates $(x, y) \mapsto(y, x)$ we may suppose that $\theta_{0} \in[0, \pi / 2)$, so that $R_{0}$ is parallel to the line $\ell: y=x \tan \theta_{0}$. Let $R^{\infty}$ be the smallest infinite strip containing $R$ and having the same slope.

Let us set

$$
B_{0}:=\left\{(x, y) \in \mathbb{Z}^{2}: \operatorname{dist}((x, y), \ell) \leq 5\right\} .
$$

In order that $R_{0}$ and $R_{\gamma}$ intersect, we need to have $|\gamma| \leq 3 N$. Thus,

$$
\sum_{\gamma \in \mathbb{Z}^{2}}\left|R_{0} \cap R_{\gamma}\right|=\sum_{\substack{\gamma \in \mathbb{Z}^{2} \cap B_{0} \\|\gamma| \leq 3 N}}\left|R_{0} \cap R_{\gamma}\right|+\sum_{\substack{\gamma \in \mathbb{Z}^{2} \backslash B_{0} \\|\gamma| \leq 3 N}}\left|R_{0} \cap R_{\gamma}\right| .
$$

As for the first term, we use a crude estimate

$$
\left|R_{0} \cap R_{\gamma}\right| \leq N \text { and } \sharp\left(B_{0} \cap\left\{\gamma \in \mathbb{Z}^{2}:|\gamma| \leq 3 N\right\}\right) \lesssim N .
$$

To bound the second term, we observe that the angle $\theta_{\gamma}$ of the long sides of $R_{\gamma}$ and $R_{0}$ always exceeds $\sin ^{-1} \frac{d_{\gamma}-1}{\sqrt{N^{2}+1}}$, where $d_{\gamma}=\frac{\left|\gamma_{2}-\gamma_{1} \tan \theta_{0}\right|}{\sqrt{1+\tan ^{2} \theta_{0}}}=\left|\gamma_{2} \cos \theta_{0}-\gamma_{1} \sin \theta_{0}\right|$ is the distance between $\gamma=\left(\gamma_{1}, \gamma_{2}\right)$ and $\ell$. Therefore,

$$
\sum_{\substack{\gamma \in \mathbb{Z}^{2} \backslash B_{0} \\|\gamma| \leq 3 N}}\left|R_{0} \cap R_{\gamma}\right| \leq \sum_{\substack{\gamma \in \mathbb{Z}^{2} \backslash B_{0} \\|\gamma| \leq 3 N}}\left|\left(R_{0}\right)^{\infty} \cap\left(R_{\gamma}\right)^{\infty}\right| \leq \sum_{\substack{\gamma \in \mathbb{Z}^{2} \backslash B_{0} \\|\gamma| \leq 3 N}} \frac{\sqrt{N^{2}+1}}{d_{\gamma}-1} \leq 4 \sum_{\substack{\gamma \in \mathbb{Z}^{2} \backslash B_{0} \\|\gamma| \leq 3 N}} \frac{N}{d_{\gamma}},
$$

where we have used Lemma 4.1. Thus

$$
\sum_{\substack{\gamma \in \mathbb{Z}^{2} \backslash B_{0} \\|\gamma| \leq 3 N}}\left|R_{0} \cap R_{\gamma}\right| \lesssim \int_{\operatorname{dist}((x, y), \ell) \geq 5, x^{2}+y^{2} \leq 9 N^{2}} \frac{N}{\left|y \cos \theta_{0}-x \sin \theta_{0}\right|} d x d y
$$




$$
\begin{aligned}
& \lesssim \int_{y \geq 5, x^{2}+y^{2} \leq 9 N^{2}} \frac{N}{|y|} d x d y \\
& \lesssim \int_{|x| \leq N, 5 \leq y \leq N} \frac{N}{y} d x d y=O\left(N^{2} \log N\right) .
\end{aligned}
$$

Thus, the estimate of the second term is valid.

\section{REFERENCES}

[1] A. Alfonseca, F. Soria And A. Vargas, A remark on maximal operators along directions in $\mathbb{R}^{2}$, Math. Res. Lett. 10 (2003), no. 1, 41-49.

[2] A. Alfonseca, F. Soria And A. VARgas, An almost-orthogonality principle in $L^{2}$ for directional maximal functions, Harmonic analysis at Mount Holyoke (South Hadley, MA, 2001), 1-7, Contemp. Math. 320, Amer. Math. Soc. Providence, RI, 2003.

[ 3 ] A. CARbery, Covering Lemmas Revisited, Proc. Edinburgh Math. Soc. (2) 31 (1988), no. 1, 145-150.

[ 4 ] A. CARbery, E. HeRnÁndeZ And F. Soria, Estimates for the Kakeya maximal operator on radial functions in $\mathbb{R}^{n}$, in Harmonic Analysis (S. Igari, ed.), ICM-90 Satellite Conference Proceedings, Springer-Verlag, Tokyo, 1991, 41-50.

[ 5 ] A. CórdobA, The Kakeya maximal function and the spherical summation multiplier, Amer. J. Math. 99 (1977), no. 1, 1-22.

[6] J. García-Cuerva And J. L. Rubio de Francia, Weighted norm inequalities and related topics, NorthHolland, Amsterdam, 1985.

[ 7 ] L. GRAFAKos, Modern Fourier Analysis, volume 250 of Graduate Texts in Mathematics. Springer, New York, 2nd edition, 2008.

[ 8 ] S. IGARI, On Kakeya's maximal function, Proc. Japan Acad. Ser. A 62 (1986), no. 8, 292-293.

[ 9 ] N. H. KATZ, Maximal operators over arbitrary sets of directions, Duke Math. J. 97 (1999), no. 1, 67-79.

[10] N. H. KATZ, Remarks on maximal operators over arbitrary sets of directions, Bull. London Math. Soc. 31 (1999), no. 6, 700-710.

[11] U. KEICH, On $L^{p}$ bounds for Kakeya maximal functions and the Minkowski dimension in $\mathbb{R}^{2}$, Bull. London Math. Soc. 31 (1999), no. 2, 213-221.

[12] D. Müller, A note on the Kakeya maximal function, Arch Math. (Basel) 49 (1987), 66-71.

[13] H. Saito And H. TANAKA, Directional maximal operator and radial weights on the plane, Bull. Aust. Math. Soc. 89 (2014), no. 3, 397-414.

[14] E. M. Stein, Harmonic Analysis, real-variable methods, orthogonality, and oscillatory integrals, Princeton University Press, Princeton, NJ, 1993.

[15] J.-O. Strömberg, Maximal functions associated to rectangles with uniformly distributed directions, Ann. Math. (2) 107 (1978), no. 2, 399-402.

[16] H. TANAKA, An elementary proof of an estimate for the Kakeya maximal operator on functions of product type, Tohoku Math. J. (2) 48 (1996), no. 3, 429-435.

ACADEMIC SUPPORT CENTER

KOGAKUIN UNIVERSITY

2665-1, NAKANOMACHI

HACHIOJI-SHI TOKYO, 192-0015

JAPAN

E-mail address: j1107703@gmail.com
DEPARTMENT OF MATHEMATICS AND

INFORMATION SCIENCES

TOKYO METROPOLITAN UNIVERSITY

1-1 MinAMi-OHSAWA

HACHIOJI-SHI TOKYO, 192-0397

JAPAN

E-mail address: ysawano@tmu.ac.jp 